\title{
Cognitiva.PT. Lectoescritura: Programa en soporte informático multimedia para la intervención en los Trastornos Específicos de la Lectoescritura.
}

\section{Manuela Torres Diez}

Fonos Salud

España 


\section{Resumen}

En este trabajo se presenta el Programa de Lectoescritura de Cognitiva.PT para la estimulación cognitiva y la rehabilitación de los Trastornos Específicos de la Lectoescritura desde una perspectiva neuropsicológica. Finalmente, se ilustra con la presentación de una programación en un caso práctico.

Palabras Clave: soporte informático multimedia, trastornos específicos de la lectoescritura.

\section{Presentación}

El Programa Lectoescritura de COGNITIVA.PT trata la estimulación cognitiva y la rehabilitación de los Trastornos Específicos de la Lectoescritura desde la perspectiva neuropsicológica, teniendo en cuenta los diversos sistemas funcionales que intervienen en ella.

En su elaboración ha intervenido un amplio equipo integrado por profesionales especialistas en diversas ramas: psicólogos, pedagogos, logopedas, informáticos y diseñadores. Esto confiere al programa solidez en la fundamentación, eficacia en la técnica y efectividad en la metodología.

El soporte informático aporta una nueva herramienta, que en algunos aspectos posibilita unsalto cualitativo en los campos del diagnóstico, habilitación y terapia de trastornos.

En ningún momento COGNITIVA.PT pretende suplantar al terapeuta, sino que, como hemos ya apuntado, es una herramienta abierta en la que éste proyectará su labor terapéutica según sus propios métodos y orientaciones. Simplemente facilitará y potenciará su trabajo.

COGNITIVA.PT cumple totalmente los requisitos que se consideran necesarios para que un programa de rehabilitación cognitiva se pueda considerar de utilidad clínica (Franco, Orihuela, Bueno y Cid, 2000):

-Flexibilidad: capacidad de adaptarse a las necesidades específicas de cada usuario.

-Sencillez: que pueda ser utilizada por el usuario con facilidad y sin precisar de la intervención continua del especialista. 
-Utilidad: que permita conseguir los objetivos terapéuticos que se pretenden y que permita la contribución clara a la mejora de las diferentes funciones superiores.

-Accesibilidad: tanto desde el punto de vista económico como de su alcance y disponibilidad en cualquier entorno.

\section{Contenidos del Programa}

Los contenidos del programa ofrecen una perspectiva integradora de los diferentes procesos que intervienen en el aprendizaje de la lectoescritura:

- Procesos perceptivo visuales (input visual).

- Procesos perceptivos auditivoverbales (input auditivo).

- Ruta fonológica o indirecta.

- Conciencia Fonológica.

- Ruta léxica o directa.

- Procesos sintácticos.

- Procesos semánticos.

\section{Potencialidad y alcance del programa}

El programa está diseñado de forma que sirva de soporte abierto a cualquier método, tendencia o sistema de trabajo, a través de las programaciones elaboradas por el propio terapeuta o incluyendo programaciones externas elaboradas por otros terapeutas y equipos.

Además, cuenta con bases de datos suficientemente amplias y estructuradas y con gran variedad de ejercicios que permite al terapeuta realizar programaciones que se ajusten a las características de los sujetos por edad y patología.

$\mathrm{Su}$ motor de inteligencia gestiona las programaciones propuestas de forma que se autoconfigura en el desarrollo de los ejercicios, adaptándose a las características del alumno, y es a su vez configurable en ciertos aspectos por el terapeuta. Esto le confiere una versatilidad extraordinaria a la hora de responder a diversos estilos de aprendizaje y terapias.

El Programa Lectoescritura de COGNITIVA.PT puede ser aplicado en dos ámbitos y con dos perspectivas complementarias: rehabilitación y estimulación o potenciación de los procesos cognitivos implicados en la lectoescritura. 


\section{Características del Programa}

El programa ofrece:

- Oportunidad de trabajar de forma independiente cada proceso, según las necesidades determinadas en el diagnóstico previo que se deberá realizar antes de proceder a la intervención.

○ Gran flexibilidad y adaptabilidad a las necesidades de cada alumno.

- Interactividad, en grado superior a cualquier otro soporte.

○ Conocimiento de resultados y tratamiento del error de forma automatizada e inmediata.

- Control de resultados, permitiendo generar informes exhaustivos de la evolución en las sesiones de rehabilitación.

- Diseño estético y funcional que facilita su uso y confortabilidad, pretendiendo en todo momento la calidad y el equilibrio en el ritmo, proporción e integración de los diversos elementos: imagen, sonido, tiempos, interactividad, etc.

- Su alto grado de autonomía permite liberar al terapeuta en gran cantidad de tareas, muchas veces tediosas por el alto grado de repetición.

\section{Descripción de los procesos cognitivos.}

El programa Lectoescritura.Cognitiva.PT., pretende plantear la rehabilitación desde una perspectiva integradora, atendiendo a los diferentes procesos cognitivos implicados en la lectoescritura. Por este motivo, se han tenido en cuenta los procesos más significativos que se han ido describiendo en los diversos modelos teóricos como el modelo modificado de Morton (1980), utilizado entre otros por Coltheart (1987), Patterson y Shewell (1987), Ellis y Young (1988), etc, (citados por Manning, 1992).

Muchas son las investigaciones que se han centrado en justificar los trastornos de la lectoescritura como dependientes de unos u otros procesos. Por ejemplo, podemos citar a autores que centraban la explicación de la dislexia como un déficit de tipo visual. Orton (1925) emitió la hipótesis de un déficit visuo-espacial. Pirozzolo (1979) y Palvidis (1981) defendían las hipótesis de anomalías en la motricidad ocular (obras citadas en Narbona y Chevrie- 
Muller, 1997), y otros autores como Lundberg y cols. (1988) y Manis y cols. (1997) (citados en Etchepareborda y Habib, 2001) han llegado a la conclusión de que el déficit central es de naturaleza fonológica.

Esta multitud de investigaciones y con conclusiones tan diversas, junto a la gran heterogeneidad de los trastornos de la lectoescritura, tanto adquiridos como evolutivos, no hacen más que indicarnos claramente que estamos intentando comprender un proceso muy complejo, por lo que el método de aprendizaje y de rehabilitación tiene que ser multimodal y abarcar todos los componentes del sistema. En el programa Lectoescritura.Cognitiva.PT. se han intentado asumir, en la medida de lo posible, las diferentes hipótesis planteadas, considerando los diferentes procesos defendidos por unos u otros autores. Esto demuestra claramente el espíritu integrador ya anteriormente señalado.

Los procesos de la lectoescritura presentados en el programa son:

1- Procesos perceptivos visuales.

2- Procesos perceptivos auditivoverbales.

3- Procesos Fonológicos (Ruta fonológica).

4- Conciencia Fonológica.

5- Procesos léxicos (Ruta léxica).

6- Procesos sintácticos.

7- Procesos semánticos.

Procesos perceptivos visuales.

Aunque hoy en día, existen muchos autores que defienden que la dislexia es un trastorno esencialmente lingüístico, y han rechazado la hipótesis de que su origen se centre en deficiencias de percepción visual, no podemos desestimar que la primera tarea del proceso de la lectura es perceptivovisual y queramos o no cumple un papel fundamental en el mismo.

En el programa no se han incluido ejercicios que se dediquen a trabajar específicamente los patrones de movimientos oculares o seguimiento visual. Partimos del sistema de análisis visual y de su relación íntima con los procesos de atención. Teniendo en cuenta que el análisis visual se debe realizar tanto de letras como de palabras, y que también depende de si las palabras son más o menos conocidas o si se trata de pseudopalabras, en el programa se barajan 
todas estas posibilidades y nos permite trabajar con letras, sílabas, palabras, pseudopalabras y frases, y seleccionar el vocabulario según su frecuencia de uso.

El modulo de ejercicios del procesamiento visual posibilita entrenar intensivamente la discriminación y reconocimiento de letras y palabras, así como los procesos de atención visual. Para la consecución de estos objetivos el programa nos permite seleccionar y modificar las diferentes variables como diversos tipos de letras, tamaños, mayúsculas y minúsculas, el tiempo de presentación de los estímulos, etc, pudiendo de esta forma elevar progresivamente el nivel de dificultad de la tarea.

La posibilidad de la modificación del tiempo de presencia del ítem visual y de los tiempos de respuesta dota al programa de una gran eficacia, y se considera requisito imprescindible si se tiene en consideración los resultados de las investigaciones que sustentan la "teoría del déficit en el procesamiento temporal" en la que se afirma que los niños disléxicos presentan una incapacidad para procesar cambios rápidos de estímulos tanto en la modalidad auditiva como visual. (Etchepareborda, 2001).

Los ejercicios que se proponen pueden configurarse y responden a los siguiente grupos:

- Identificación y comparación según modelo

- Correspondencias

- Identificación dentro de un grupo

- Identificación dentro de un texto

- Identificación palabras intrusas

- Asociar letras por pauta-clave

- Responder a un estímulo

Procesos perceptivos auditivos.

Existen teorías, como hemos comentado en el apartado anterior, que defienden que los trastornos de la lectoescritura son consecuencia de un déficit sensorial, tanto visual como auditivo. Concretamente en las investigaciones de Tallal y Piercy (1973) (obra citada en Etchepareborda, 2002, y en Soriano-Ferrer, 2004) apoyan el déficit en el procesamiento temporal de los estímulos auditivos. Demostraron que los niños con trastornos del lenguaje oral y escrito tenían deficiencias en el procesamiento de estímulos auditivo presentados de forma rápida 
y sucesiva. Bajo estos supuestos teóricos, en el programa se proponen ejercicios de atención, discriminación y memoria auditiva y fonológica.

El objetivo del módulo es el entrenamiento intensivo en ejercicios de atención y discriminación de estímulos auditivoverbales, con el fin de superar o mejorar las deficiencias del procesamiento. Las unidades de discriminación en este módulos son: tonos puros, fonemas, sílabas y palabras. No se ha considerado oportuno incluir ruidos y sonidos no verbales (como sonidos realizados con objetos, sonidos producidos con el cuerpo, sonidos de animales,...) tan frecuentes en los programas de discriminación auditiva, porque no tienen una relación directa con el procesamiento fonológico, ni con el aprendizaje de la lectoescritura.

El programa facilita el entrenamiento intensivo en discriminación y memoria auditiva con tonos puros y series de tonos controlando la presentación de los estímulos en diferentes frecuencias, y permitiendo pasar de discriminaciones de tonos que se perciben como distintos claramente hasta llegar a tonos con frecuencias muy próximas.

Otra opción que tiene el programa, que puede resultar muy interesante para la rehabilitación, es la posibilidad de presentar los estímulos auditivos por ambos oídos simultáneamente, por uno u otro o con intensidad distinta en cada uno de ellos. Aunque en las investigaciones sobre especialización hemisférica y lenguaje oral que se ha trabajado con escucha dicóti$c a$, es decir, presentando dos estímulos auditivos distintos simultáneamente a cada oído, y también con presentaciones monoaurales, se ha llegado a conclusiones contradictorias sobre la dominancia de un oído u otro, ya que dependerá de diversos factores y más concretamente del tipo de estímulo auditivo que se trate (Junqué y Barroso, 1999), poder controlar y comprobar el rendimiento del usuario del programa dependiendo de que el estímulo se presente de forma biaural o monoaural puede resultar una estrategia con un gran potencial en la rehabilitación.

Teniendo en cuenta la propia experiencia clínica en la intervención de trastornos fonológicos tanto en el lenguaje oral como escrito, hemos considerado que era necesario que el programa contara con ejercicios para el entrenamiento en discriminación e identificación de los fonemas puros, puesto que los niños con trastornos fonológicos tiene muchas dificultades de discriminar y aislar el fonema en la unidad silábica. En este módulo se prepara y se empieza a trabajar la consolidación y automatización de la correspondencia fonema-grafema. El entrenamiento se iniciará con la presentación multimodal de los fonemas puros (sonidoimagen articulatoria-grafema/s) para llegar a su integración en las sílabas de diferentes estruc- 
turas y posteriormente de las sílabas en las palabras. El terapeuta controlará las variables de tiempo de presentación y respuesta, fonemas-grafemas de trabajo, estructuras silábicas y longitud de la palabras para adaptar la programación a las necesidades de cada usuario e ir modificándolas conforme a sus progresos.

Para facilitar la discriminación fonológica el programa permite modificar la presentación de la palabra modelo para la realización del ejercicio mediante la repetición de la sílaba objetivo, así como modificando el volumen de su presentación, y este mismo refuerzo también se utiliza para el tratamiento del error, además también de posibilitar la presentación secuenciada de las diferentes unidades: fonema puro, sílaba y palabra.

Los ejercicios que se proponen pueden configurarse y responden a los siguiente grupos:

- Discriminación, memorización, localización y reproducción de tonos.

Para la realización de los ejercicios de este módulo se dispone de una base de datos con 60 tonos monofónicos, 28 acordes (tonos polifónicos) y 28 arpegios (varios tonos). Se puede seleccionar el número de tonos que componen el ejercicio de 2 a 5 tonos y el rango de frecuencias de trabajo (graves/medias/agudas/totalidad de tonos) lo que permite ir aumentando progresivamente la complejidad. Pudiéndose además trabajar con tonos aislados como con series de tonos.

En los ejercicios de discriminación se presentan dos o más tonos o series de tonos y el sujeto tiene que identificar si son iguales, reconocer el tono más grave o el tono más agudo. Para la realización del ejercicio se puede elegir trabajar con discriminación de sonidos monofónicos, arpegios ascendentes, arpegios descendentes y acordes.

En los de memorización se presenta un tono modelo y el sujeto debe recordar que tono era entre un grupo de tonos presentados posteriormente. Se puede seleccionar el mismo tipo de sonidos que en los ejercicios de discriminación.

- Discriminación, memorización, asociación y reproducción de fonemas-grafemas.

En este módulo se trabaja con la presentación de fonemas puros aislados o series de fonemas puros. El terapeuta puede seleccionar los fonemas que desea trabajar según los problemas específicos que haya detectado, o seguir un programa de general de estimulación que se inicie con la discriminación entre pares de fonemas muy distantes hasta llegar a los fone- 
mas más próximos, como por ejemplo los fonemas /R/L/D/ que suelen plantear muchas dificultades de discriminación.

Los ejercicios de discriminación consisten en escuchar fonemas o series de fonemas e identificar cuales de ellos son iguales.

En los de memorización se presenta un fonema o serie de fonemas como modelo y luego se debe recordar los que son iguales al modelo entre los fonemas presentados posteriormente en forma secuencial o simultánea.

En los ejercicios de asociar se presenta como modelo un fonema o serie de fonemas (presentación auditiva) o un grafema o serie de grafemas (presentación visual) y el sujeto debe reconocer si los fonemas o grafemas que se presentan posteriormente corresponden con el modelo.

Y por último, los ejercicios de reproducir son ejercicios de dictado de fonemas o series de fonemas, es decir, sujeto escucha un fonema y debe escribirlo utilizando el teclado.

En los puntos posteriores se pueden realizar los mismos tipos de ejercicios que aquí se han descrito pero trabajando con sílabas o palabras.

- Discriminación, memorización, asociación y reproducción de sílabas.

Para la realización de los ejercicios de sílabas se cuenta con una base de ++++ sílabas que mediante la pantalla de configuración se pueden seleccionar con facilidad los fonemas dentro de la sílaba y las estructuras silábicas. De tal forma que podemos trabajar la discriminación de sílabas tanto por contraste entre fonemas (ej. /P-B/, /S-Z/,...) como por permutación del orden de los fonemas (ej.: $\mathrm{CV} / \mathrm{VC}, \mathrm{CCV} / \mathrm{CVC}$ ) o por número de fonemas que componen la sílaba (ej.: CV/CVC, $\mathrm{CVC} / \mathrm{CVCC}, \ldots)$.

- Discriminación, memorización, asociación y reproducción de palabras.

El programa dispone de una base de datos de ¿??? Palabras. El programa permite seleccionar con rapidez las palabras a trabajar eligiendo el fonema y estructura silábica que deseamos.

- Discriminación, memorización, asociación y reproducción de pares mínimos de palabras por oposición fonológica. 
- Asociación fonema/sílaba, fonema/palabra y sílaba/palabra.

El apartado de asociación nos permite trabajar la discriminación e identificación de un fonema en una sílaba o palabra. Se presenta un fonema y el sujeto debe de identificar cuales de las sílabas o palabras presentadas posteriormente contiene dicho fonema. De la misma forma se trabaja con las sílabas, es decir tras presentar una sílaba modelo se seleccionará que palabra o palabras contiene dicha sílaba.

Procesos Fonológicos (Ruta Fonológica).

La ruta fonológica se basa en la recuperación del sonido que corresponde a cada grafía (transcodificación grafema-fonema) y nos permite leer palabras nuevas y pseudopalabras. Este mecanismo se relaciona directamente con los procesos fonológicos del habla, afirmación que se confirma al comprobar que los niños con trastornos fonológicos manifiestan dificultades en la ruta fonológica de la lectura y cometen errores principalmente por sustitución, alteración del orden y omisión de fonemas, omisiones de nexos de palabras y de los comienzos y finales de las palabras.

Para optimizar la ruta fonológica, en primer lugar, hay que superar las dificultades de discriminación fonológica, si las hubiera, para proceder posteriormente a la automatización de la relación fonema-grafema de tal forma que permita una lectura rápida sin errores. Según se mejora en este proceso se produce un progreso simultáneo en la comprensión lectora e integración de la información, puesto que se libera al sistema del esfuerzo que supone la atención en los niveles inferiores de procesamiento, y los recursos cognitivos se pueden dedicar a las funciones superiores.

Este módulo, por tanto, está totalmente relacionado con el anterior, y se deberán trabajar de forma complementaria y sucesiva. El objetivo de este apartado será llegar a la automatización de la asociación grafema-fonema que permitirá una lectura rápida y sin errores.

Los ejercicios propuestos en este módulo son:

- Ejercicios con sílabas:

- lectura de sílabas con fonemas-grafemas contrastados.

- lectura de sílabas por

- reconocer las combinaciones de letras que son sílabas posibles de nuestro idioma. 
- Ejercicios con palabras:

- lectura de palabras que contienen sílabas propuestas.

- lectura de palabras con iluminación de consecutiva de las sílabas.

- seleccionar los grafemas que forman una palabra oída previamente.

- seleccionar las sílabas que forman una palabra oída previamente.

- escritura de los grafemas que faltan en una palabra escrita de la palabra oída

- escritura de las sílabas que faltan en una palabra escrita de la palabra oída.

- Ejercicios con frases/textos:

- lectura de frases/textos con iluminación de sílabas.

- lectura de frases/textos con iluminación de palabras.

\section{Conciencia Fonológica.}

Bermeosolo (1994) define la conciencia fonológica como "un proceso cognitivo complejo que permite ejecutar operaciones mentales sobre el out del mecanismo de percepción del habla”. (citado en Etchepareborda, 2001). El desarrollo de la conciencia fonológica implica la toma de conciencia de la existencia de los fonemas como unidades mínimas que componen las sílabas y las palabras y la habilidad para operar con ellas. En los últimos años las investigaciones sobre la conciencia fonológica y su relación con el aprendizaje de la lectoescritura han sido muy numerosas y fructíferas y se ha llegado a un cierto consenso sobre la importancia de esta habilidad para la adquisición de la lectura. En cambio , todavía está abierto el debate sobre su carácter predictivo, puesto que para algunos autores como Goswami (1991) aparece como un prerrequisito para dicho aprendizaje, y en cambio para otros autores como de Morais y cols. (1987) se considera que existe una interacción simultánea en el tiempo, es decir, la conciencia fonológica se va adquiriendo al mismo tiempo que la lectura, no la precede (obras citadas en Narbona y Chevrie-Muller, 1997).

En el desarrollo y estudio de este aspecto han tenido mucho peso los enfoques psicolingüísticos de la lectura, desde los cuales se defiende la idea de que la lectura constituye una actividad lingüística secundaria, que depende del lenguaje oral, entendido como la actividad lingüística primaria. Así pues, el buen lector, será el que emplee sus competencias lingüísticas eficazmente tanto para la comprensión y producción del lenguaje oral como del lenguaje escrito (Escoriza, 1990). Además, se entiende que el lenguaje no es solo un instrumento de co- 
municación sino un objeto de conocimiento, proponiéndose desde este momento el desarrollo de las habilidades metalingüísticas, donde quedará incluida la conciencia fonológica.

En este módulo se proponen ejercicios con el fin de conseguir que el usuario llegue a conocer las diferentes unidades lingüísticas (fonema, sílaba, palabra) y domine las habilidades de análisis-síntesis con dichas unidades. El método instruccional que se sigue va de lo concreto a lo abstracto. Por tanto, se iniciará con la unidad lingüística de la palabra, que resulta más significativa, y seleccionando el vocabulario más familiar, puesto que en los resultados de diferentes investigaciones se ha concluido que la identificación de las diferentes unidades lingüísticas está facilitada por el significado, hasta llegar a las tareas de análisis-síntesis con la unidad más abstracta que es el fonema y con vocabulario no familiar (Escoriza, 1992). En todas las actividades se podrá presentar la palabra oral, su imagen (en las actividades de vocabulario frecuente), la palabra escrita y las unidades fonema, sílaba y palabra (por medio de símbolos, grafemas y sílabas) para dar un formato concreto y visual a las unidades abstractas y auditivas. Con las distintas unidades se realizarán ejercicios de análisis que consistirán básicamente en supresión, conteo, identificación e inversión de segmentos y ejercicios de síntesis que suponen la integración de los distintos elementos segmentados hasta llegar a la unidad de sílaba y palabra.

A continuación enumeramos los tipos de ejercicios propuestos:

- Ejercicios para el desarrollo del conocimiento léxico:

- segmentación léxica oral.

- segmentación léxica escrita.

- Ejercicios para el desarrollo del conocimiento silábico:

- contar el número de sílabas de una palabra.

- reconocer que queda de una palabra cuando se omite una sílaba en posición inicial, media, final.

- identificación de la sílaba inicial, media y final de un palabra presentada con su imagen.

- identificar que sílaba ocupa un lugar determinado en una palabra presentada con su imagen.

- identificación de las palabras presentadas con imágenes tiene sílabas comunes. 
- reconocer la palabra pronunciada con segmentación silábica y asociarla con su palabra escrita o su imagen.

- pronunciar una palabra invirtiendo el orden de las sílabas.

- identificación de la sílaba tónica de una palabra.

- Ejercicios el desarrollo del conocimiento fonémico:

- contar el número de letras de una sílaba.

- contar el número de letras de una palabra.

- reconocer la palabra que ha sido presentada por medio de deletreo.

- asociar la estructura vocálica con su palabra correspondiente.

- identificar las palabras que tienen un fonema/grafema dado en diferentes posiciones.

- identificar las palabras que riman con una palabra dada.

- identificar la letra que ocupa un lugar señalado en una palabra.

\section{Procesos Léxicos.}

Actualmente parece que se ha superado la controversia sobre cual es el método (global o analítico) más eficaz para el aprendizaje de la lectura, llegándose a la conclusión de la necesidad de la complementariedad de los dos tipos de enfoques, puesto que en el proceso de la lectoescritura se utilizan indistintamente ambas estrategias. Cuando se está trabajando desde una perspectiva global se está desarrollando la ruta léxica o directa, ya que se está enriqueciendo el almacén del léxico visual, mientras que el método analítico refuerza la asociación fonema-grafema. Según Vellutino y Scanlon (1989) el método global se basa en el significado, presentando las palabras como unidades no segmentables, almacenando la imagen global de la palabra para acceder directamente a su representación lexical o semántica (citado en Escoriza y Boj, 1992).

A través de la ruta léxica se leen las palabras familiares y las palabras extranjeras que se han introducido en nuestro idioma, puesto que si se leen utilizando las reglas de transformación grafema-fonema no se podrá acceder a su significado.

Con este módulo se pretende aumentar el léxico visual y de mejorar el acceso al léxico mediante ejercicios de presentación de palabras de diferentes campos semánticos, tareas de discriminar homófonos, diferenciar palabras de pseudopalabras... Cuanto mayor es el almacén 
de léxico visual, más se utiliza la ruta directa o léxica en la lectura, aumentando considerablemente la velocidad lectora, al mismo tiempo que también disminuirán las faltas de ortografía arbitraria.

Los ejercicios propuestos en este módulo son los siguientes:

- Agrupar, seleccionar, asociar, identificar palabras y su categoría.

- Formar palabras con una serie de sílabas propuestas.

- Reconocer la palabra presentada con sílabas desordenadas.

- Identificar las palabras reales en un grupo de palabra y pseudopalabras.

- Palabras derivadas.

- Asociar la pronunciación de la palabras pseudoextranjeras con su forma escrita.

- Lectura a velocidad creciente de palabras, empezando por el vocabulario frecuente.

- Búsqueda y conteo de palabras concretas en un texto.

- Identificar las palabras que cumplen una consigna en una serie de palabras presentadas en velocidad progresiva.

\section{Procesos Sintácticos.}

El procesamiento léxico (comprensión de las palabras) es una condición necesaria, pero no suficiente para llegar a la comprensión de un texto. Las frases se componen por palabras relacionadas según unas reglas sintácticas, y solo se llega a la comprensión de las mismas cuando se dominan esas relaciones. El lector a través del procesamiento sintáctico asigna funciones a los distintos componentes de la oración, especificando la relación entre ellos. Según Vieiro (2003) el procesamiento sintáctico permite al lector la utilización de las siguientes estrategias: a) establecer el orden de las palabras; b) establecer la relación entre las palabras contenido y las palabras función; c) atribuir el significado a las palabras y d) elaborar estructuras sintácticamente diferentes gracias a los signos de puntuación.

Los ejercicios que presenta el programa para el entrenamiento en el procesamiento sintáctico son:

- Ordenar frases presentadas con palabras desordenadas.

- Comprensión de frases con diferentes estructuras gramaticales mediante la asociación de frases a dibujos. 
- Cambiar el significado de las frases mediante el uso de los signos de puntuación.

- Lectura y signos de puntuación.

Procesos Semánticos.

El procesamiento semántico es fundamental para que tenga lugar la comprensión del texto y se realiza a través de los siguientes subprocesos: extracción del significado, interrelación del significado en los conocimientos previos y el nivel de conocimientos del lector. Supone, por tanto, que la extracción del significado de las palabras se tiene que coordinar con el significado que se desprende de las estructuras sintácticas y con los conocimiento previos, tanto del tema en concreto que se trata en el texto como de las estructuras y esquemas propios de organización interna de los textos.

Los ejercicios propuestos en este módulo son:

- Ejercicios con frases:

- Lectura comprensiva de órdenes.

- Completamiento de frases (ejercicios tipo cloze).

- Identificar absurdos verbales.

- Identificar la causa/consecuencia en una frase.

- Identificar la respuesta correcta a una pregunta planteada.

- Dada una respuesta identificar la pregunta correspondiente.

- Asociar frases que tiene el mismo significado.

- Ejercicios con textos:

- Ordenación temporal de frases para formar relatos.

- Organización de textos.

- Palabras desaparecidas. Procedimiento cloze.

- Entrenamiento de los esquemas de las historias y textos.

- Análisis de párrafos. Búsqueda de la idea principal.

- Formulación de preguntas sobre el texto.

- Formular hipótesis. Realiza predicciones sobre lo que ocurrirá en la lectura.

- Poner título al texto. 
- Búsqueda de información concreta del texto. Preguntas literales e inferenciales.

- Deducción del significado de palabras a partir del contexto.

\section{Modalidades o Vías de Navegación del Programa Cognitiva. PT}

El Programa Lectoescritura de COGNITIVA.pt presenta 4 modalidades:

\section{Modalidad Alumno}

- Gestiona la sesión de trabajo del alumno.

\section{Modalidad Tutor}

- Gestión de los datos del alumno (identificación, altas, etc.)

- Asignar las programaciones a utilizar para cada alumno.

- Adaptar aspectos de estas programaciones.

- Realizar informes de sesión del alumno.

- Crear copias de seguridad de los resultados obtenidos.

\section{Modalidad Programador}

- Permite realizar programaciones y baterías de ejercicios, según los diversos criterios, tendencias y métodos terapéuticos.

- Estas programaciones quedarán a disposición del tutor que las aplicará a cada alumno partiendo de sus necesidades y objetivos.

\section{Modalidad Examen diagnóstico}

- Posibilita la aplicación directa de ejercicios, configurados en el momento, con el fin de delimitar la línea base al inicio de tratamiento. 


\section{Diagrama de Funcionamiento del Programa de Lectoescritura de Cognitiva. PT}

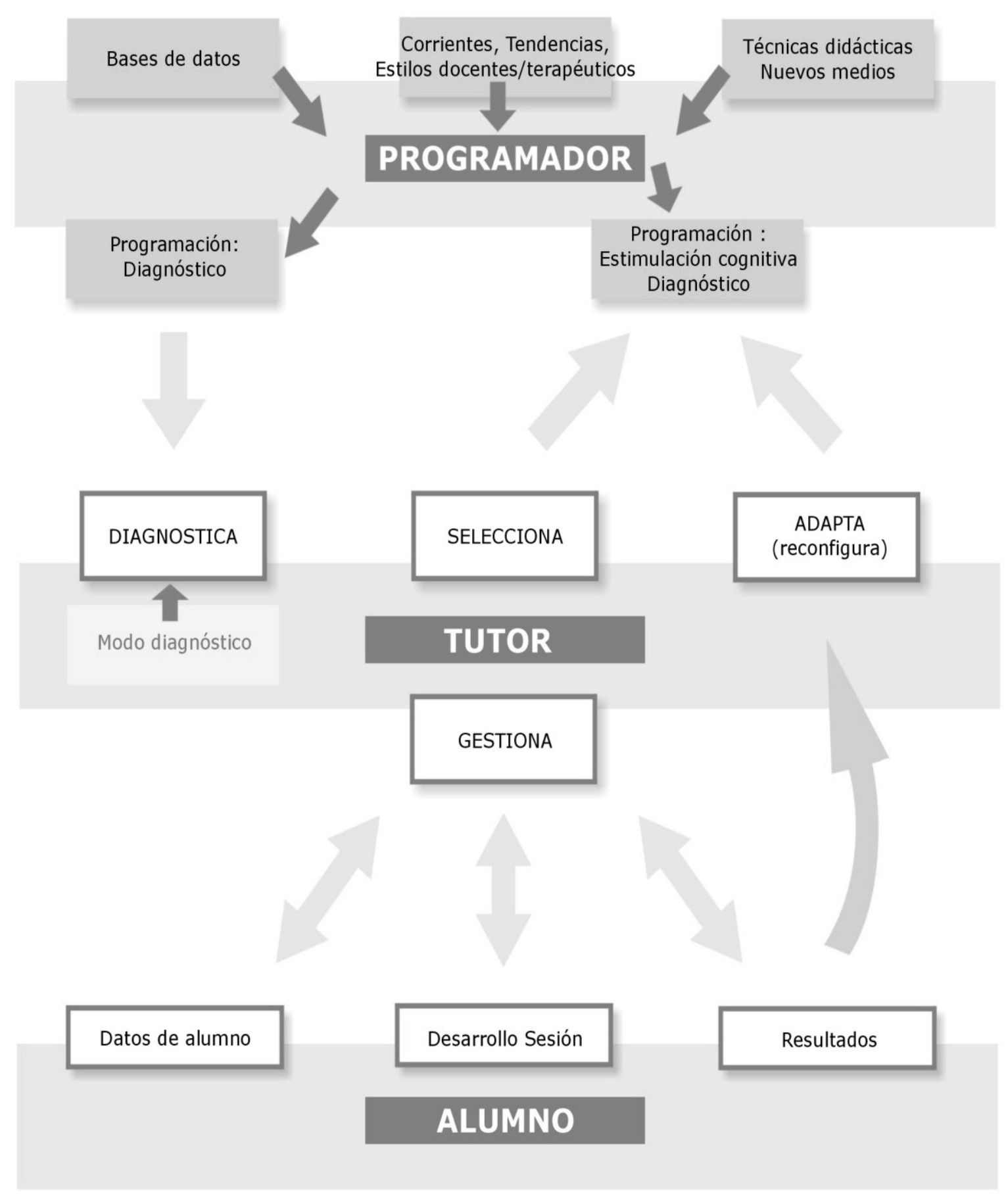




\section{Caso Práctico: Desarrollo de una programación para la intervención en un Trastorno}

\section{Fonológico.}

\section{Presentación del caso}

- Sujeto: varón de 6 años, 10 meses.

- Lengua materna: castellano.

- Bilingüísmo: no.

- Curso escolar: $1^{\circ}$ de Primaria.

- Motivo de consulta: dificultades en el aprendizaje de la lectoescritura.

- Resumen de la anamnesis: desarrollo normal en todas las áreas excepto en el lenguaje. Inicio del habla algo tardío (20 meses) con posterior evolución rápida, aunque con dificultades de pronunciación. Todavía no ha superado totalmente los problemas de habla. Le cuesta pronunciar palabras largas. Nunca ha recibido tratamiento logopédico.

Valoración diagnóstica. Pruebas y exámenes realizados.

- Escala de Inteligencia de Weschsler para Niños Revisada. WISC-R.

- Examen de órganos articulatorios y praxias orofaciales.

- Exploración fonética.

- Discriminación fonológica con lista de pares mínimos de QUILIS.

- Registro Fonológico Inducido.

- Prueba de Evaluación de la Discriminación Auditiva y Fonológica. EDAF.

- Subprueba "Percepción Auditiva" de la Batería BADYG-Gráfico.

- Prueba de Segmentación Lingüística. PSL.

- Test de Análisis de la Lectura y Escritura. T.A.L.E.

- Registro y análisis de lenguaje espontáneo.

Tras el análisis de los resultados obtenidos en las distintas pruebas se obtienen las siguientes conclusiones: el niño posee una capacidad cognitiva media (CI verbal $=112$, CI manipulativo $=110$ ). Tiene adquiridos todos los fonemas y estructuras silábicas del castellano, pero se registran dislalias fonológicas por sustitución, omisión y alteración del orden de los fonemas, principalmente en las palabras polisílabas, palabras con estructuras silábicas complejas y palabras no familiares. Aunque las sustituciones entre fonemas es bastante asistemá- 
tica, las sustituciones más frecuentes se han dado entre "LL-Ñ", "P-B-M", "R-L-D". Tiene un rendimiento bajo en las tareas de discriminación, integración, memoria y conciencia fonológica. Presenta claramente dificultades en el aprendizaje de la lectoescritura. No tiene adquirida la lectura de las estructuras silábicas inversas, mixtas, sinfones vibrantes y líquidos. Su lectura de palabras y frases es muy lenta, silabeante y con numerosos errores por omisión, sustitución y alteración del orden de las letras, este mismo tipo de errores los comete en la escritura.

El juicio diagnóstico ha sido de Trastorno fonológico, que se manifiesta tanto en el lenguaje oral como escrito. Por tanto, sus problemas en el aprendizaje de la lectoescritura son consecuencia del déficit fonológico.

\section{Programación desarrollada con COGNITIVA.PT.Lectoescritura.}

El programa de intervención se desarrollará siguiendo cuatro fases:

\section{$1^{\text {a }}$ Fase. Estimulación general:}

Este caso precisa entrenar de forma intensiva la atención, discriminación y memoria auditiva en términos generales, que se realizará con los ejercicios de tonos. De esta manera estamos potenciando estas capacidades que se consideran como prerrequisitos indispensables para cualquier tarea posterior que tenga como ínput un estímulo auditivo como es el caso del análisis fonológico que se debe llevar a cabo tanto en el lenguaje oral como escrito. En estas primeras sesiones el objetivo será la estimulación de la discriminación fonológica y visual con todos los fonemas y grafemas, y con las estructuras silábicas más frecuentes, dejando las sílabas más complejas e infrecuentes y los grupos consonánticos para otra fase posterior.

\section{$2^{a}$ Fase. Entrenamiento específico:}

Se centrará en el trabajo intensivo de la discriminación fonológica y visual de los fonemas-grafemas de sustitución más frecuente, así como los procesos fonológicos referidos a la estructura silábica (reducción y alteración del orden de fonemas-grafemas) que también son más numerosos.

Los fonemas-grafemas que sustituye de forma más sistemática son: "Ñ-LL", "P-B-M" y "R-L-D", y esto se da tanto en el lenguaje oral como escrito. En cambio, las omisiones en las sílabas mixtas terminadas con el fonema o grafema "S" y la sustitución entre sílabas mixtas con los grafemas "L-R" y los sinfones correspondientes se da casi exclusivamente en la lecto- 
escritura. Así que en la programación se trabajarán con mucha intensidad estos procesos fonológicos y faltas de ortografía natural.

\section{$3^{\text {a }}$ Fase. Entrenamiento avanzado:}

En este punto del programa se trabajará las estructuras silábicas más complejas (CCVC, CVCC, VCC, CCVC) e infrecuentes, y la discriminación e integración fonológica en palabras polisílabas e infrecuentes. El objetivo es conseguir ampliar y consolidar su almacén de léxico fonológico, de tal forma que con este sobreentrenamiento aumentará la frecuencia y uso de las palabras y se reducirá considerablemente el porcentaje de errores tanto en el lenguaje oral como escrito.

\section{$4^{\text {a }}$ Fase. Afianzamiento y automatización:}

En esta última fase del tratamiento se programación sesiones con todos los fonemas y estructuras silábicas anteriormente trabajadas, y tanto con vocabulario frecuente e infrecuente. En este punto los objetivos serán reducir al máximo el número de errores y conseguir un aumento progresivo de la velocidad. Para alcanzar estos objetivos se manipularán las siguientes variables: reducción del tiempo de presentación de los estímulos y el tiempo de respuesta, tamaño de las letras, tipos de grafía, aumento del número de elementos del ejercicio, eliminación de los modelos y reducción progresiva de las ayudas.

\section{Referencias}

Artigas-Pallarés, J. (2002). "Problemas asociados a la dislexia". Rev. Neurol., vol. 34, supl.1, S7-S13.

Borzone, A.M.(1984). "Fonética experimental y psicolingüística”. Rev. Logop. Fonoaud.,vol. IV, $\mathrm{n}^{\mathrm{o}} 1,37-51$

Bosch, L. y Herrero, T. (1988). "Discriminación auditiva y análisis de los componentes fónicos de las palabras". Rev. Logop. Fonoaud., vol. VIII, n 3, 134-139.

Castaño, J.(2002). “Aportes de la neuropsicología al diagnóstico y tratamiento de los trastornos de aprendizaje". Rev. Neurol., vol. 34, supl.1, S1-S7.

Correig, M. y Jiménez, I.(1989): "Papel de la fonología en la preparación de los niños para la lectura y escritura". Rev. Logop. Fonoaud., vol. IX, no 4, 200-207.

Cuetos, F., Rodríguez, B., y Ruano, E. (1996). PROLEC. Batería de evaluación de los procesos lectores de los niños de Educación Primaria. Madrid: TEA. 
Del Río, D. y López-Higes, R. (2003). "Especificidad del área de Broca en la comprensión de oraciones". Rev. Logop. Fonoaud., vol. 23, n 3, 154-163.

Escoriza, J.(1990). “Origen y supuestos básicos del enfoque psicolingüístico de la lectura”. Rev. Logop. Fonoaud., vol. X, no 4, 192-200.

Escoriza, J., y Barberán, C. (1992).“Intervención educativa a nivel de comprensión del principio alfabético (I)”. Rev. Logop. Fonoaud., vol. XII, nº 1, 25-31.

Escoriza, J., y Barberán, C. (1992).“Intervención educativa a nivel de comprensión del principio alfabético (II)”. Rev. Logop. Fonoaud., vol. XII, nº 2, 104-110.

Etchepareborda, M.C. (2002). "Detección precoz de la dislexia y enfoque terapéutico". Rev. Neurol., vol.34, supl. 1, S13-S23.

Etchepareborda, M.C. y Habib, M.(2001). "Bases neurobiológicas de la conciencia fonológica: su compromiso en la dislexia". Rev. Neurol. Clin., vol. 2 (1), 5-23.

Franco, M., Orihuela, T., Bueno Y. y Cid. T. (2000). Programa Gradior. Programa de evaluación y rehabilitación cognitiva por ordenador. Valladolid: Edintras.

Fernández, M. y Ramos, F.(1991). "El aprendizaje de la lectura. Reflexión sobre la validez de los modelos explicativos actuales". Rev. Logop. Fonoaud., vol. IX, nº 2, 197-203.

Junqué, C.(1992). "La neuropsicología clínica: evolución de una especialidad”. Rev. Logop. Fonoaud., vol. XII, n $3,134-137$.

Junqué, C. y Barroso, J. (1999). Neuropsicología. Madrid: Síntesis.

Junqué, C., Bruna, O. y Mataró, M. (2003). Neuropsicología del lenguaje. Funcionamiento normal y patológico. Rehabilitación. Barcelona: Masson.

Lozano, A., Ramírez, M. y Ostrosky-Solís, F. (2003). "Neurobiología de la dislexia del desarrollo: una revisión”. Rev. Neurol., vol. 36, n 11, 1077-1082.

Manning, L. (1992). Introducción a la neuropsicología clásica y cognitiva del lenguaje. Teoría, evaluación y rehabilitación de la afasia. Madrid: Trotta.

Martín, M.P. La lectura. Procesos neuropsicológicos de aprendizaje, dificultades, programas de intervención y estudio de casos. Barcelon: Lebón.

Molina, S., Sinués, A., Deaño, M., Puyuelo, M., y Bruna, O.(1998). El fracaso en el aprendizaje escolar (II). Dificultades específicas de tipo neuropsicológico. Málaga: Ajibe.

Muñoz, M.D. (2003). Rehabilitación de la lengua oral y escrita. Bases neuropsicolingüísticas. Madrid: CEPE.

Narbona, J. y Chevrie-Muller, C. (1997). El lenguaje del niño. Desarrollo normal, evaluación y trastornos. Barcelona: Masson.

Nespoulous, J., Roch, A. y Puel, M.(1982). "Lingüística y semiología afásica”. Rev. Logop. Fonoaud., vol. II, nº 4, 79-90.

Nicasio, J. (1998). Manual de dificultades de aprendizaje. Lenguaje, Lecto-Escritura y Matemáticas. Madrid: Narcea. 
Parkin, A. (1999). Exploraciones en neuropsicología cognitiva. Madrid: Médica Panamericana.

Peña Casanova, J. y Barraquer Bordas, L. (1982). "Sobre la 'representación' del lenguaje en el cerebro". Rev. Logop. Fonoaud., vol. I, no 3, 132-150.

Peña-Casanova, J.(1991). Programa integrado de exploración neuropsicológica. "Test Barcelona”. Normalidad, semiología y patologías neuropsicológicas. Barcelona: Masson.

Puyuelo, M. y Rondal, J.A. (2003). Manual de desarrollo de alteraciones del lenguaje. Aspectos evolutivos y patología en el niño y en el adulto. Barcelona: Masson.

Quintana, L.(1988). "La lectura en clase: ejercicios concretos para un objetivo específico". Rev. Logop. Fonoaud., vol VIII, nº 1, 2-10.

Recasens, M.(1992). "Velocidad y comprensión lectoras: recursos didácticos". Rev. Logop. Fonoaud., vol. XII, n 1, 19-24.

Rodríguez, M.C. (1990). "Consideraciones sobre los inicios de la escritura" Rev. Logop. Fonoaud., vol. X, n $2,66-73$.

Solé, I.(1989). "La enseñanza de la lectura a lo largo de la escolaridad". Rev. Logop. Fonoaud., vol. IX, nº 4, 208-215.

Soriano-Ferrer, M. (2004). "Implicaciones educativas del déficit cognitivo de la dislexia evolutiva”. Rev. Neurol., vol. 38,supl 1, S47-S52.

Vallés, A. (1998). Dificultades de aprendizaje e intervención psicopedagógica. Valencia: Promolibro. 